\title{
PLANET FORMATION
}

\section{Cloudy, with highs of 1,200 degrees}

A protoplanet has been directly imaged in the gap in the transitional disk around pre-main sequence dwarf star PDS 70 (pictured) using the SPHERE instrument on the Very Large Telescope (VLT). This discovery is reported in two papers to be published in Astronomy \& Astrophysics:

Keppler et al. (preprint at https://arxiv.org/ abs/1806.11568) and Müller et al. (preprint at https://arxiv.org/abs/1806.11567).

Typically, the presence of gaps in protoplanetary disks alone is used to infer the presence of forming planets, although in recent years there have also been directly observed signs of objects embedded in young disks that may be linked to nascent planets. However, neither gaps themselves, disk features (such as spirals or azimuthal asymmetries or shadowed regions) nor the embedded objects seen to date are robust signs of planet formation. This detection of protoplanet PDS 70b with SPHERE is claimed to be the first sound detection of such a young planet.

SPHERE works in the optical and nearinfrared $(0.65-2.32 \mu \mathrm{m})$, and employs a coronagraph mask (represented by the black circle in the centre of the image) and a polarimetric differential imaging technique to obtain the high-contrast imaging required to directly detect planets in faint disks. Utilizing the high angular resolution of the VLT, SPHERE observed a bright point source within a gap in the dusty transitional disk at a radius of $\sim 22$ au (projected separation; 195 milliarcsec) over five different epochs. An orbit at this distance would take $\sim 120$ years to complete, and indeed the point source was seen to move slightly between epochs. The point source was confirmed using the VLT's NaCo instrument and by re-reducing archival Gemini L'-band data taken in 2012. The $~ 54$-au-wide gap in the transition disk between an inner disk

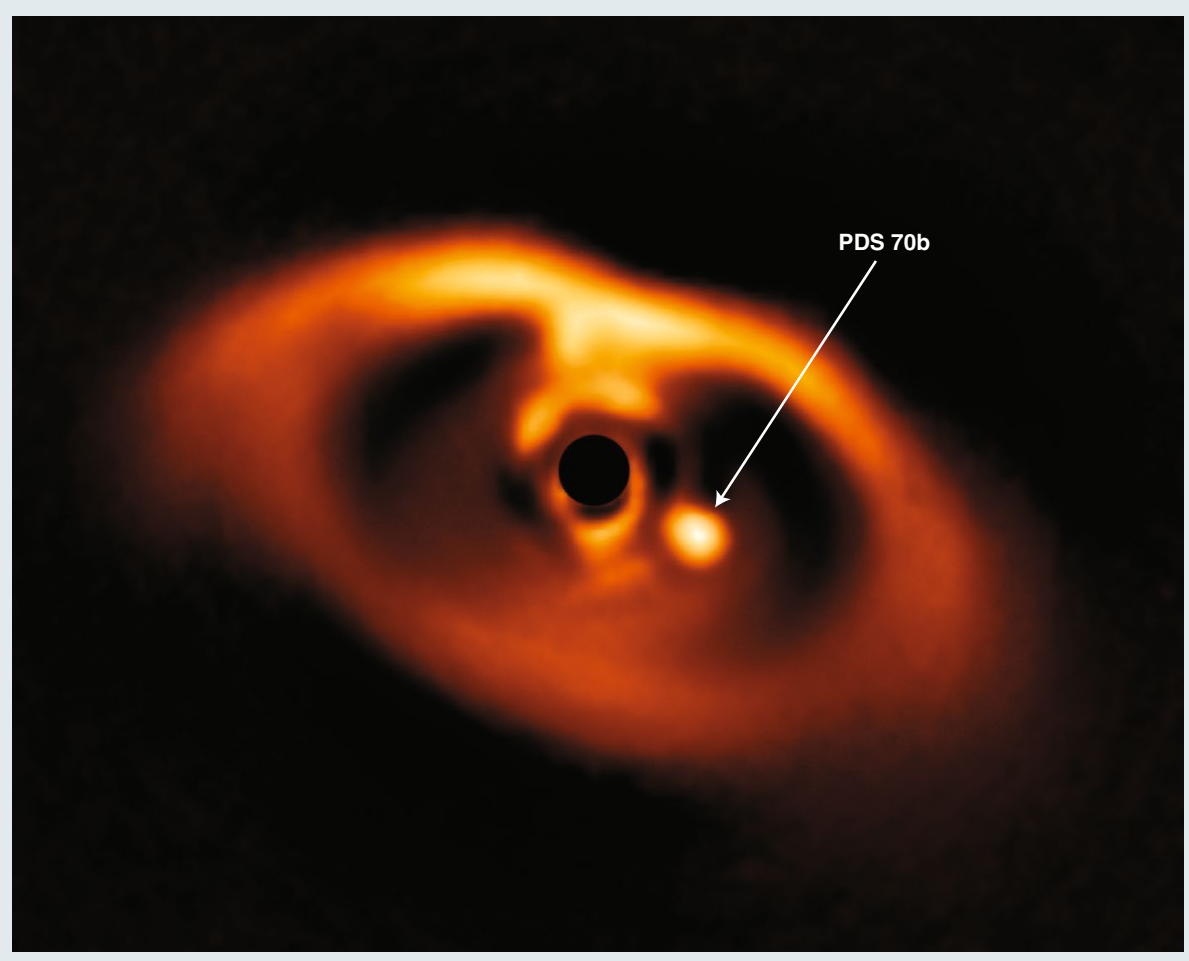

Credit: ESO/A. Müller et al

and an outer ring was seen previously and confirmed by these new observations.

Comparing the infrared colours of PDS 70b to measured and synthetic photometry of red, M, L and T dwarfs, and planets, Keppler et al. find that PDS $70 \mathrm{~b}$ is quite unusual, appearing redder than most known dusty (or cloudy) companion bodies. Comparing the infrared colours to synthetic planetary evolutionary tracks suggests a mass for the planet of 5-14 $M_{\text {Jup }}$, depending on assumptions, and comparing the spectral energy distribution of PDS 70b to a radiative model gives a minimum radius for the planet of $1.3 R_{\text {Iup }}$ and an effective temperature of $\sim 1,200 \mathrm{~K}$. Müller et al. extend this analysis, taking spectra of the young planet from $\sim 1-1.7 \mu \mathrm{m}$ with SPHERE, and comparing the results to different 1D cloudy and cloud-free atmospheric models. They conclude that the spectrum and additional photometry of PDS 70b is best fit by a planet of radius 1.4-3.7 $R_{\text {Jup }}$ and a cloudy atmosphere model.

\section{Paul Woods}

Published online: 1 August 2018 https://doi.org/10.1038/s41550-018-0552-7 